\title{
Enhancement of spin Hall effect induced torques for current-driven magnetic domain wall motion: Inner interface effect
}

\author{
Do Bang, ${ }^{1,2}$ Jiawei Yu, ${ }^{3}$ Xuepeng Qiu, ${ }^{3}$ Yi Wang, ${ }^{3}$ Hiroyuki Awano, ${ }^{1}$ Aurelien Manchon, ${ }^{4}$ and Hyunsoo Yang ${ }^{3}$ \\ ${ }^{1}$ Toyota Technological Institute, Tempaku, Nagoya 468-8511, Japan \\ ${ }^{2}$ Institute of Material Science, Vietnam Academy of Science and Technology, 18 Hoang Quoc Viet, Cau Giay, Ha Noi, Vietnam \\ ${ }^{3}$ Department of Electrical and Computer Engineering, National University of Singapore, 117576, Singapore \\ ${ }^{4}$ Division of Physical Science and Engineering, King Abdullah University of Science and Technology (KAUST), \\ Thuwal 23955, Saudi Arabia
}

(Received 3 February 2015; revised manuscript received 31 March 2016; published 23 May 2016)

\begin{abstract}
We investigate the current-induced domain wall motion in perpendicular magnetized $\mathrm{Tb} / \mathrm{Co}$ wires with structure inversion asymmetry and different layered structures. We find that the critical current density to drive domain wall motion strongly depends on the layered structure. The lowest critical current density $\sim 15 \mathrm{MA} / \mathrm{cm}^{2}$ and the highest slope of domain wall velocity curve are obtained for the wire having thin Co sublayers and more inner $\mathrm{Tb} / \mathrm{Co}$ interfaces, while the largest critical current density $\sim 26 \mathrm{MA} / \mathrm{cm}^{2}$ required to drive domain walls is observed in the $\mathrm{Tb}-\mathrm{Co}$ alloy magnetic wire. It is found that the $\mathrm{Co} / \mathrm{Tb}$ interface contributes negligibly to Dzyaloshinskii-Moriya interaction, while the effective spin-orbit torque strongly depends on the number of $\mathrm{Tb} / \mathrm{Co}$ inner interfaces $(n)$. An enhancement of the antidamping torques by extrinsic spin Hall effect due to $\mathrm{Tb}$ rare-earth impurity-induced skew scattering is suggested to explain the high efficiency of current-induced domain wall motion.
\end{abstract}

DOI: 10.1103/PhysRevB.93.174424

\section{INTRODUCTION}

The current-induced domain wall motion (CIDM) in thin perpendicular magnetized ferromagnetic wires sandwiched between a heavy metal and an oxide has been shown to be very efficient, yielding a high domain wall (DW) velocity at low current density enabling the development of spintronic devices [1-12]. In these asymmetric wires, inversion symmetry breaking together with strong spin-orbit interaction (SOI) has two major implications. First, it induces spin-orbit torques (SOTs) due to, on the one hand, the interfacial Rasba effect and, on the other hand, the spin Hall effect (SHE) present in the bulk heavy metal [13-17]. Both effects produce fieldlike and antidampinglike torques [18-20]. Second, it is also responsible for the emergence of interfacial DzyaloshinskiiMoriya interaction (DMI), which promotes chiral magnetic textures. The concurrence of antidamping SOT and DMIinduced chiral textures results in fast DW motion in ultrathin layer structures [21-28].

Recently, a transition in the mechanism responsible for current-driven DW motion from bulk (spin-transfer) to interfacial (spin-orbit) torque has been confirmed as a change in the DW motion direction when reducing the layer thickness of $\mathrm{Co} / \mathrm{Ni}$ multilayer down to $2.1 \mathrm{~nm}$ [29]. It is found that the adiabatic spin-transfer torque (STT) dominates the DW motion in the thick regime, while the antidamping SOT controls the DW motion in the thin regime. In an earlier study [30], on the other hand, SOTs-induced DW motion was observed up to a thickness of $\sim 10 \mathrm{~nm}$ for the asymmetric interfacial wire with the layered structure of $\mathrm{SiO}_{2} /[\mathrm{Tb}(3.2 \AA) / \mathrm{Co}(2.6 \AA)]_{\mathrm{n}} / \operatorname{Pt}(20 \AA)$. The high efficiency of SOTs in the wire can be attributed to an enhancement of antidamping SOT by inner Tb/Co interfaces. However, such an enhancement in thick multilayers has not yet been fully understood.

In this study, we present the current-induced DW motion in the $\mathrm{Tb} / \mathrm{Co}$ wires with different layered structures. The magnitude of the antidamping SOT by inner $\mathrm{Co} / \mathrm{Tb}$ interfaces is tuned by changing the thickness of Co ultrathin sublayers, number of inner $\mathrm{Co} / \mathrm{Tb}$ interfaces, and formation of $\mathrm{Tb}-\mathrm{Co}$ alloy magnetic layer. Our results show that the efficiency of SOTs is highest for the samples with a larger number of inner $\mathrm{Co} / \mathrm{Tb}$ interfaces and lowest for the one with $\mathrm{Tb}-\mathrm{Co}$ alloy magnetic wire. DMI is found to be small in our samples. We suggest that the high efficiency of SOTs in our multilayers can be associated with extrinsic SHE due to Tb rare-earth impurityinduced skew scattering at inner $\mathrm{Co} / \mathrm{Tb}$ interfaces [31,32].

\section{SAMPLE PREPARATION AND MAGNETIC PROPERTIES}

For this work, experiments are performed on three asymmetric magnetic wires with different layer structures [Fig. 1(a)], in which the total magnetic layer thickness is kept to be $\sim 6 \mathrm{~nm}$. Layers of A stack: $[\mathrm{Tb}(3.4 \AA) /$ $\mathrm{Co}(3.2 \AA)]_{9} / \operatorname{Pt}(20 \AA) ; \quad \mathrm{B} \quad$ stack: $[\mathrm{Tb}(3.4 \AA) / \operatorname{Co}(6.5 \AA)]_{6} /$ $\operatorname{Pt}(20 \AA)$; and $\mathrm{C}$ stack: [Tb ${ }_{45} \mathrm{Co}_{55}$ alloy $\left.(60 \AA)\right] / \operatorname{Pt}(20 \AA)$ are deposited on the thermally oxidized silicon substrates by $\mathrm{dc}$ and $\mathrm{rf}$ magnetron sputtering using high-quality $\mathrm{Tb}$ and Co targets. The base pressure of the growth chamber is $\sim 5 \times 10^{-8}$ mbar. The Ar $(99.99 \%)$ gas pressure is kept at $\sim 3 \times 10^{-2}$ mbar during sputtering. The growth rates of $\mathrm{Tb}$ and Co sublayers are 0.56 and $0.53 \AA / \mathrm{s}$, respectively. Therefore, it is expected that only a part of the first Tb sublayer is oxidized due to the minimal residual oxidation contamination from the $\mathrm{SiO}_{2} / \mathrm{Si}$ substrate. The patterned wires have a width of $1.1 \mu \mathrm{m}$ and are fabricated by using electron-beam lithography and lift-off technique. The motion of DWs in the wires is driven by pulses of voltage between two Al/Ti electrodes, labeled as (1) and (2) in Fig. 1(b), and directly observed using polar Kerr microscopy [33]. All measurements shown in this work are performed at room temperature. Figure 1(b) shows the image of a scanning electron microscope (SEM) with the schematic 
(a)
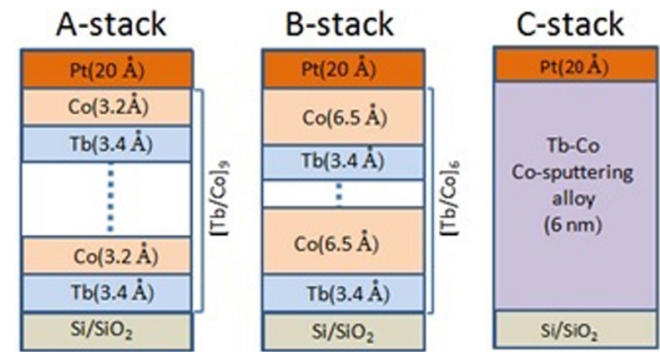

(b)

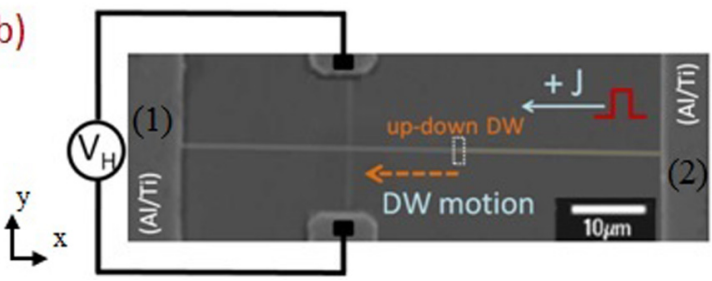

FIG. 1. (a) Schematic illustration of multilayered $\mathrm{Tb} / \mathrm{Co}$ films with structural inversion asymmetry. (b) Scanning electron microscopy image of multilayered $\mathrm{Tb} / \mathrm{Co}$ wire with $\mathrm{Ti} / \mathrm{Al}$ electrodes for magnetotransport measurements.

electrical measurement setup. Both the film and patterned wires exhibit perpendicular magnetic anisotropy (PMA), which is confirmed by using an alternative gradient force magnetometer (AGFM) and anomalous Hall effect (AHE) measurements.

Figure 2(a) shows the hysteresis loops of the three magnetic films with different layered structures when the field is applied normal to the film plane, indicating that the films have perpendicular magnetic anisotropy. However, the magnetic films show different values of the coercivity $\left(H_{\mathrm{c}}\right)$ and saturation magnetization $\left(M_{\mathrm{s}}\right)$. The uniaxial anisotropy constant $\left(K_{\mathrm{u}}\right)$ is determined to be $1.44 \times 10^{5}, 2.8 \times 10^{5}$, and $1.6 \times 10^{5} \mathrm{~J} / \mathrm{m}^{3}$ from the $M_{\mathrm{s}}$ and saturation field $\left(H_{\mathrm{k}}\right)$ of in-plane (hard axis) hysteresis loops (not shown) for the A-, B-, and C-stack films, respectively. These different magnetic properties can be attributed to the different Tb-Co compositions, where the A stack has a rare-earth (RE)-rich composition, while $\mathrm{B}$ and $\mathrm{C}$ stacks have transition-metal (TM)-rich compositions, which can be qualitatively determined from the polarity of Kerr rotation angle from these films. In multilayers, $\mathrm{Tb}$ and $\mathrm{Co}$ moments are seen to be in the homogeneous antiferromagnetic state $[34,35]$. Therefore, the direction of the total magnetic moment in the whole stack depends on the moments of the $\mathrm{Tb}$ and Co sublattices: in a stack with Tb-rich composition, the moment of $\mathrm{Tb}$ sublattice is larger than that of the Co sublattice, while in a stack with Co-rich composition, the moment of Co sublattice is larger than that of the Tb sublattice. When the composition of the whole stack changes from Co rich to $\mathrm{Tb}$ rich, the direction of the total magnetic moment can be detected by a polarity change in the Kerr rotation angle.

Typical results for DW depinning field measurements for the three magnetic wires are shown in Fig. 2(b). As the out-of plane magnetic field changes from 0 to $-2.5 \mathrm{kOe}$, the normalized Hall voltage $\left(V_{\mathrm{H}}\right)$ changes from 1 to -1 indicating that the magnetization is reversed in the Hall cross and the DW passes through the Hall cross region. Here, we define the DW depinning field $\left(H_{\text {dep }}\right)$ as the magnetic field where $V_{\mathrm{H}}$ starts reducing from 1 . Furthermore, it is found that $H_{\text {dep }}$

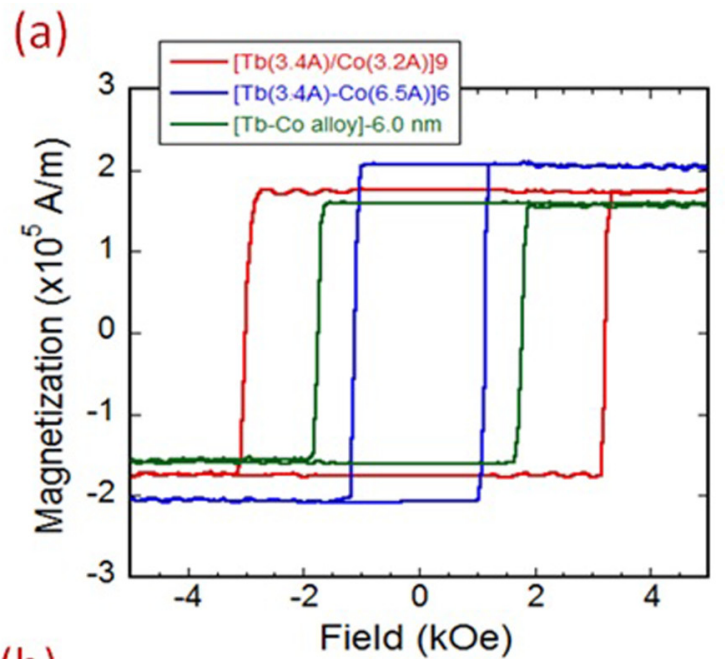

(b)

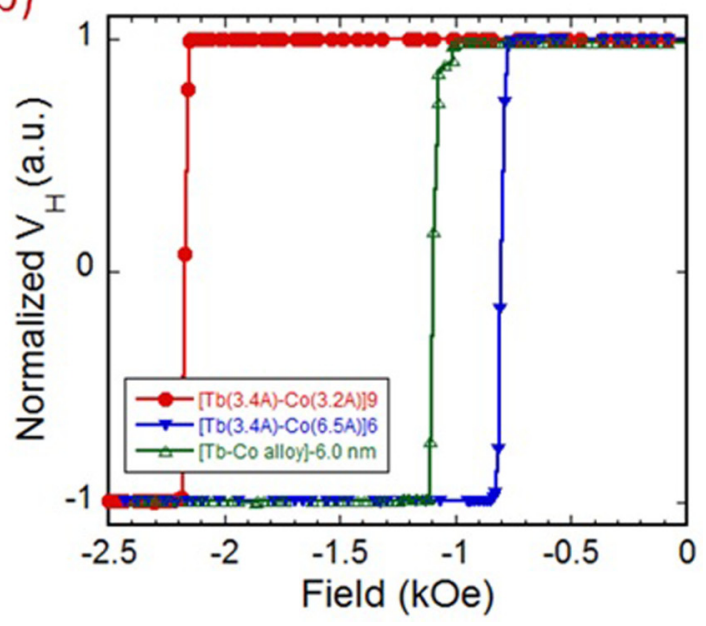

FIG. 2. (a) Magnetization curves of multilayered Tb/Co films. (b) Normalized anomalous Hall voltage as a function of the perpendicular field measured after the creation of a DW for different stack structures: A, B, and C stacks.

increases as the wire width of the A stack is reduced as shown in the inset of Fig. 3(a). The error bas was determined from five repeated measurements. It is clear that $H_{\text {dep }}$ shows a linear dependence on 1/width. Similar to the study of $\mathrm{Co} / \mathrm{Ni}$ nanowires with perpendicular magnetic anisotropy [36], this size dependence of $H_{\text {dep }}$ indicates that extrinsic pinning dominates field-induced DW motion in the wire.

\section{CURRENT-DRIVEN DOMAIN WALL MOTION}

\section{A. Critical current density}

We next investigate current-induced DW motion by applying voltage pulses of 100-ns duration between (1) and (2) electrodes. Figure 3(a) shows the critical current density $\left(J_{\mathrm{c}}\right)$ as a function of $H_{\text {dep }}$ for the A-stack wire. Here, $J_{\mathrm{c}}$ is determined as the minimum current density required to drive DW with a depinning field of $H_{\text {dep }}$. The experiment was also repeated five times for each data point. Similar to a previous study [30], the DW moves along the direction of current flow $(+J)$ in our asymmetric interfacial wires. This reversed DW motion with respect to electrons is explained by the concurrence of 


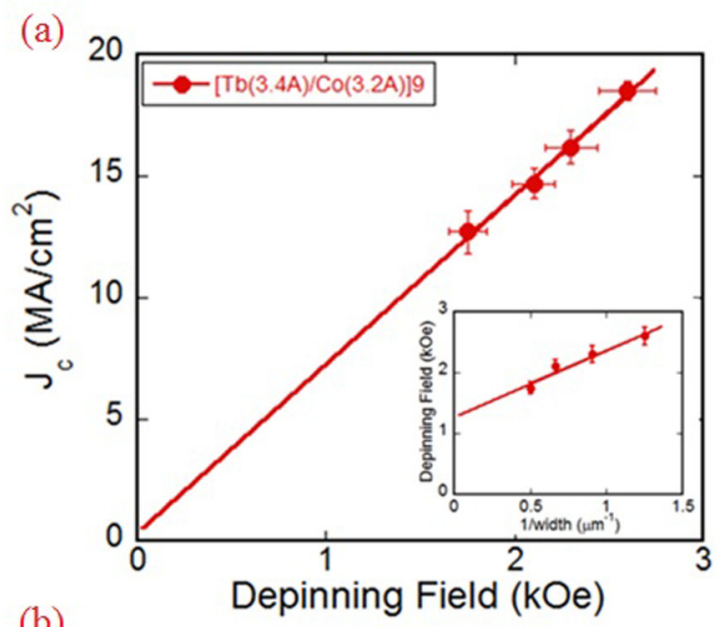

(b)
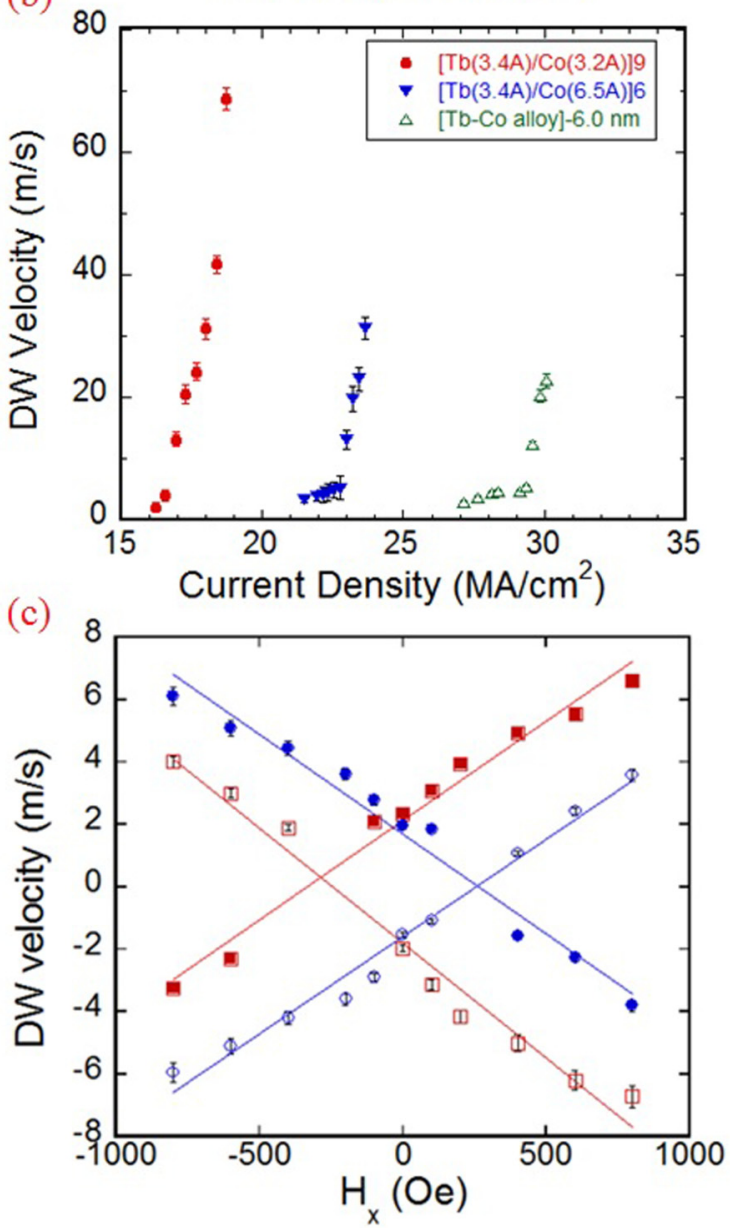

FIG. 3. (a) Critical current density $\left(J_{\mathrm{c}}\right)$ as a function of depinning field $\left(H_{\mathrm{dep}}\right)$ for the different widths of the A-stack wire. The inset shows the wire width dependence of depinning field. Solid lines are the linear fits. (b) Current density dependence of DW velocity for different magnetic wires (1.1- $\mu \mathrm{m}$ width) of A-, B-, and C-stack structures. (c) Measured DW velocity versus in-plane field $\left(H_{\mathrm{x}}\right)$ for A-stack wire. Red and blue symbols represent $\downarrow \uparrow$ and $\uparrow \downarrow$ DWs, respectively. Solid and open symbols correspond to positive and negative current directions, respectively. Lines are linear fits to the data to estimate crossing fields.

antidamping torque and DMI. It is clear that $J_{\mathrm{c}}$ is linearly proportional to $H_{\text {dep }}$. This observation suggests that current- induced antidamping SOT can be regarded as an effective depinning field $[9,15,25,37,38]$. The contribution of antidamping SOT has been added to the Landau-Lifshitz-Gilbert equation to describe the time evolution of the magnetization by Haazen et al. [37]. Their results indicate that the $H_{\text {dep }}$ can be tuned as a linear function of the current. Moreover, Emori et al. [9] also has showed that the current generates an effective field associated with a Slonczewski-like torque. The magnitude of the effective field induced by the current is estimated to be $\sim 500$ and $\sim 2000$ Oe per $10^{12} \mathrm{~A} . \mathrm{m}^{-2}$ for the $\mathrm{Pt} / \mathrm{CoFe} / \mathrm{MgO}$ and $\mathrm{Ta} / \mathrm{CoFe} / \mathrm{MgO}$ nanowires, respectively.

In order to study the effect of the layered structure on the current-induced DW motion, we have measured the DW velocity $(v)$ as a function of the injected current density $(J)$ for A, B, and C stacks, as sketched in Fig. 1(a). The results are shown in Fig. 3(b) for a wire width of $1.1 \mu \mathrm{m}$. $J_{\mathrm{c}}$ strongly increases for the wires of the B stack and $\mathrm{C}$ stack. In addition, the slope of $v$ versus $J$ curves gradually reduces in these wires. The highest slope was obtained for A-stack wire where there are more interfaces between $\mathrm{Tb}$ and $\mathrm{Co}$, while the lowest slope was seen for the $\mathrm{C}$-stack wire with a thick $\mathrm{Tb}_{45} \mathrm{Co}_{55}$ alloy magnetic layer.

\section{B. Dzyaloshinkii-Moriya interaction}

The effect of a longitudinal field $\left(H_{\mathrm{x}}\right)$ on the DW velocity is shown in Fig. 3(c) for A-stack wire (measured at 15.2 MA $/ \mathrm{cm}^{2}$ ). Red and blue symbols represent $\downarrow \uparrow$ and $\uparrow \downarrow$ DWs, and solid and open symbols correspond to positive and negative current directions, respectively. One can see that DW velocity for both $\downarrow \uparrow$ and $\uparrow \downarrow$ DWs vanishes at a certain value of $H_{\mathrm{x}}\left(H_{\mathrm{cr}} \approx \pm 265 \mathrm{Oe}\right)$, which is associated with the DMI field [25].

To evaluate the effect of the number of inner $\mathrm{Tb} / \mathrm{Co}$ interfaces on the DMI, we have measured the DMI constant and DMI effective field for the A stack with different repetition numbers ( $n=5$ and 6). The field-induced asymmetric DW expansion was investigated using a polar Kerr microscope, and the measurement schematic is presented in Fig. 4(a). The magnetic field was applied in plane with a small out-of-plane tilting $\left(\theta=5^{\circ}\right)$ to move the DW. As shown in Fig. 4(b), the DWs on the left edge of the circular domain moves faster than that on the right edge, indicating a left-handed Néel wall, which is stabilized by the presence of the DMI field [37,39]. We investigated systematically the DW velocities as a function of external field strength (not shown) then extracted DMI field as discussed in Ref. [40]. The extracted DMI constants (D) are shown at the bottom of each picture in Fig. 4(b), with the $\mathrm{Co} / \mathrm{Tb}$ bilayer repetition numbers $(n)$. We found that the extracted DMI field is comparable with the crossing field $\left(H_{\mathrm{cr}}\right)$, which is estimated from Fig. 3(c). One can see in Fig. 4(b) that the strength of DMI reduces with increasing $n$ (i.e., with increasing the overall film thickness). This indicates that DMI in these films is controlled largely by the $\mathrm{Co} / \mathrm{Pt}$ top interface.

\section{Spin-orbit torque measurement}

Using Hall voltage and lock-in measurements [41,43], both longitudinal $\left(H_{\mathrm{L}}\right)$ and transverse $\left(H_{\mathrm{T}}\right)$ SOT effective fields were estimated for the A-stack sample as shown in Fig. 4(c). It is reported in previous works [17,41,42] that 
(a)

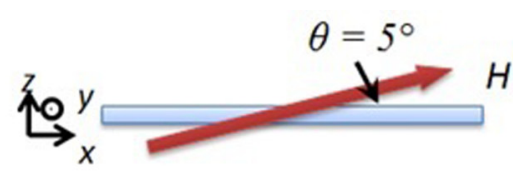

(b)
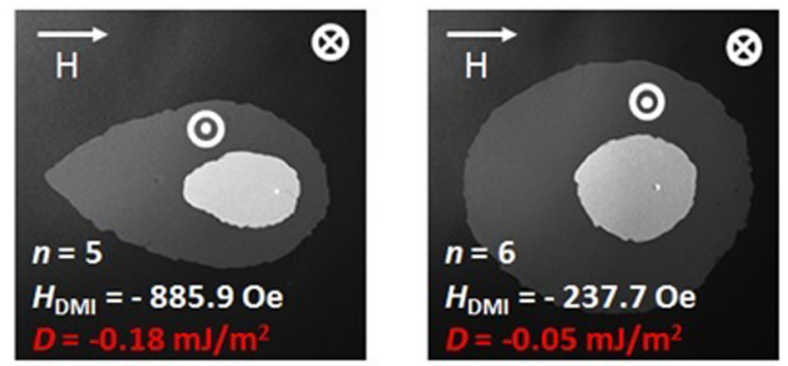

(c)

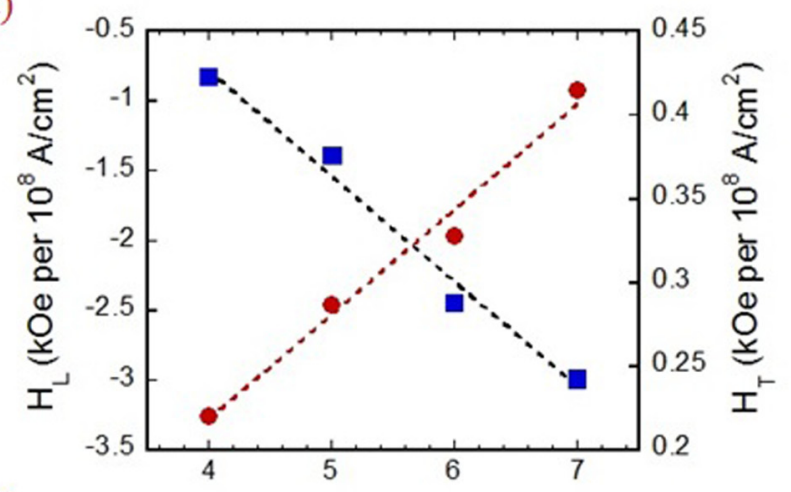

(d)

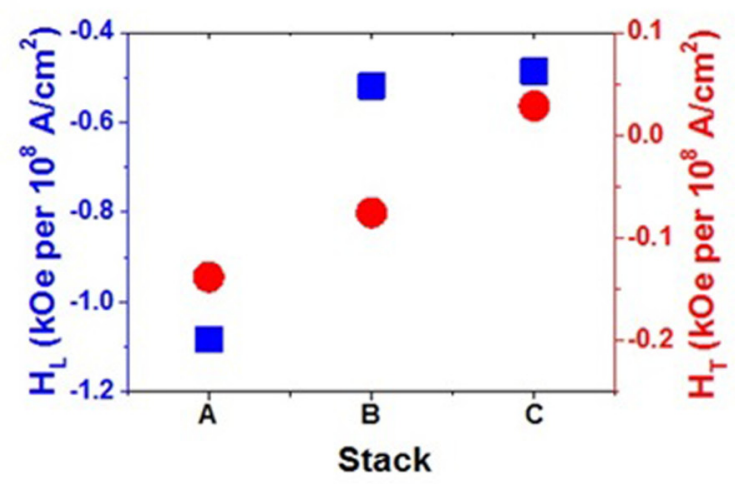

FIG. 4. (a) The lateral view of the DMI measurement schematic in the Kerr microscope. The blue horizontal box indicates the film and the red arrow presents the direction of the external field. (b) Anisotropic domain wall displacement under an in-plane magnetic field with a small out-of-plane angle for the A stack with repetition number $n=5$ and 6 . The magnetic field direction is indicated at the top of each picture. The initial domain position is indicated by the white area. (c) The dependence of longitudinal $\left(H_{\mathrm{L}}\right)$ and transverse $\left(H_{\mathrm{T}}\right)$ SOT effective fields on the number of repetitions $n$ for the A-stack sample. (d) The $H_{\mathrm{L}}$ and $H_{\mathrm{T}}$ in A-, B-, and C-stack samples. In (c),(d), the square symbols are for $H_{\mathrm{L}}$, and the circle symbols are for $H_{\mathrm{T}}$.

SOT is purely an interfacial effect, inversely proportional to the film thickness. In our case, however, SOT increases with the number of interfaces, which strongly suggests significant contribution from the $\mathrm{Co} / \mathrm{Tb}$ interfaces of the film. Our large SOT may be compared to those of Jamali et al. [43] and Lee et al. [44], who found large effective fields with a $[\mathrm{Co} / \mathrm{Pd}]_{22}$ mutilayered system and a bulk perpendicular magnetic anisotropy $\mathrm{Pd} / \mathrm{FePd} / \mathrm{MgO}$ system, respectively.

The SOT effective fields in A, B, and C stacks were also investigated as presented in Fig. 4(d). The largest SOT effective fields $\left(H_{\mathrm{L}}\right.$ and $\left.H_{\mathrm{T}}\right)$ are found in the A stack, which has the largest number of $\mathrm{Co} / \mathrm{Tb}$ interfaces $(n=9)$. The SOT effective fields are found to be minimum in the $\mathrm{C}$ stack, which contains no $\mathrm{Co} / \mathrm{Tb}$ interface $(n=0)$. The increase of SOT effective fields in Fig. 4(d) as well as the DW velocity in Fig. 3(b) with respect to the $\mathrm{Co} / \mathrm{Tb}$ interface strongly indicates the contribution of SOTs from the inner $\mathrm{Co} / \mathrm{Tb}$ interface. It is noted that $H_{\mathrm{T}}$ is about one order of magnitude smaller than $H_{\mathrm{L}}$. This suggests that the antidamping torque, possibly arising from SHE, is dominant at $\mathrm{Co} / \mathrm{Tb}$ inner interfaces.

\section{INTERPRETATION AND DISCUSSION}

\section{A. Thickness dependence of DMI and SOT}

In Sec. III, we found that the DMI field decreases when increasing the number of repetition in the A stack, whereas the SOT effective fields increase. We now propose a toy model to interpret these behaviors. In magnetic multilayers, each magnetic layer $i$ is embedded between two interfaces (top and bottom). Due to spin-orbit effects a current-driven SOT effective field is created at these two interfaces,

$$
\begin{aligned}
& \vec{h}_{\mathrm{SO}}^{\text {bottom }}=h_{\perp}^{b, i} \vec{y}+h_{\|}^{b, i} \vec{y} \times \vec{m}_{i} \\
& \vec{h}_{\mathrm{SO}}^{\text {top }}=-h_{\perp}^{t, i} \vec{y}-h_{\|}^{t, i} \vec{y} \times \vec{m}_{i},
\end{aligned}
$$

where $h_{\perp, \|}^{\alpha, i}$ is the (out-of-plane and in-plane) SOT field at the interface $\alpha$, and we chose the orientation $\vec{y}=\vec{z} \times \vec{j}_{c} /\left|\vec{z} \times \vec{j}_{c}\right|$ to comply with the symmetry of the system, without assuming any specific mechanism (spin Hall or inverse spin galvanic effects). Here, $\vec{m}_{i}$ is the magnetization direction of layer $i$ and the minus sign "-" in Eq. (2) emphasizes the fact that in the case layer $i$ is embedded between symmetric interfaces, the current-driven SOT fields on both interfaces are equal and opposite. Hence, the torque on layer $i$ reads

$$
\vec{\tau}_{i}=\vec{m}_{i} \times\left[\left(h_{\perp}^{b, i}-h_{\perp}^{t, i}\right) \vec{y}+\left(h_{\|}^{b, i}-h_{\|}^{t, i}\right) \vec{y} \times \vec{m}_{i}\right] .
$$

The same reasoning applies to the DMI field $\vec{H}_{\mathrm{DM}}^{i}$ felt by layer $i$ (see, e.g., Ref. [38]),

$$
\vec{H}_{\mathrm{DM}}^{i}=\delta\left(h_{\mathrm{DM}}^{b, i}-h_{\mathrm{DM}}^{t, i}\right) \vec{m}_{i} \times\left[(\vec{z} \times \vec{\nabla}) \times \vec{m}_{i}\right] .
$$

Here, $\delta$ is the domain wall width.

Let us now consider the stack $\mathrm{SiO}_{2} /[\mathrm{Tb} / \mathrm{Co}]_{\mathrm{n}} / \mathrm{Pt}$, composed of a repetition of $n$ bilayers. We assume that the magnetic layers composing the stack, Co and $\mathrm{Tb}$, are antiferromagnetically aligned and can be treated as macrospins, forming a perfect ferrimagnet, i.e., $\vec{m}_{\mathrm{Co}}^{j}=-\vec{m}_{\mathrm{Tb}}^{i}=\vec{m}$. The total magnetization is therefore,

$$
\begin{gathered}
\vec{M}=\sum_{i} M_{s}^{\mathrm{Tb}, i} \vec{m}_{\mathrm{Tb}}^{i}+\sum_{j} M_{s}^{\mathrm{Co}, j} \vec{m}_{\mathrm{Co}}^{j}=M_{s} \vec{m}, \\
M_{s}=(n-1)\left(M_{s}^{\mathrm{Co}}-M_{s}^{\mathrm{Tb}}\right)+M_{s, \mathrm{Top}}^{\mathrm{Co}}-M_{s, \mathrm{Bottom}}^{\mathrm{Tb}} .
\end{gathered}
$$


After some straightforward algebra, we obtain the Landau-Lifshitz-Gilbert (LLG) equation of the multilayer stack

$$
\partial_{t} \vec{m}=-\gamma \vec{m} \times\left(\vec{H}+\Delta H_{\mathrm{DM}}^{\mathrm{eff}}[(\vec{z} \times \vec{\nabla}) \times \vec{m}]\right)+\alpha_{\mathrm{eff}} \vec{m} \times \partial_{t} \vec{m}+\vec{m} \times\left[h_{\perp}^{\mathrm{eff}} \vec{y}+h_{\|}^{\mathrm{eff}} \vec{y} \times \vec{m}\right] .
$$

In Eq. (7), we have defined the following effective DMI field, magnetic damping, and SOT fields,

$$
\begin{aligned}
& H_{\mathrm{DM}}^{\mathrm{eff}}=\left[M_{s, \text { Bottom }}^{\mathrm{Tb}}\left(h_{\mathrm{DM}}^{\mathrm{SiO}_{2} / \mathrm{Tb}}-h_{\mathrm{DM}}^{\mathrm{Tb} / \mathrm{Co}}\right)+(n-1)\left(M_{s}^{\mathrm{Tb}}-M_{s}^{\mathrm{Co}}\right)\left(h_{\mathrm{DM}}^{\mathrm{Co} / \mathrm{Tb}}-h_{\mathrm{DM}}^{\mathrm{Tb} / \mathrm{Co}}\right)+M_{s, \mathrm{Top}}^{\mathrm{Co}}\left(h_{\mathrm{DM}}^{\mathrm{Tb} / \mathrm{Co}}-h_{\mathrm{DM}}^{\mathrm{Co} / P t}\right)\right] / M_{s} \\
& \alpha_{\mathrm{eff}}=\alpha\left(M_{s, \text { Bottom }}^{\mathrm{Tb}}+(n-1) M_{s}^{\mathrm{Tb}}+M_{s, \mathrm{Top}}^{\mathrm{Co}}+(n-1) M_{s}^{\mathrm{Co}}\right) / M_{s} \\
& h_{\perp}^{\mathrm{eff}}=\left[-M_{s, \mathrm{Bottom}}^{\mathrm{Tb}}\left(h_{\perp}^{\mathrm{Tb} / \mathrm{Co}}-h_{\perp}^{\mathrm{SiO} / \mathrm{Tb}}\right)+M_{s, \mathrm{Top}}^{\mathrm{Co}}\left(h_{\perp}^{\mathrm{Tb} / \mathrm{Co}}-h_{\perp}^{\mathrm{Co} / P t}\right)+(n-1)\left(M_{s}^{\mathrm{Co}}-M_{s}^{\mathrm{Tb}}\right)\left(h_{\perp}^{\mathrm{Tb} / \mathrm{Co}}-h_{\perp}^{\mathrm{Co} / \mathrm{Tb}}\right)\right] / M_{s} \\
& h_{\|}^{\mathrm{eff}}=\left[M_{s, \text { Bottom }}^{\mathrm{Tb}}\left(h_{\|}^{\mathrm{Tb} / \mathrm{Co}}-h_{\|}^{\mathrm{SiO}_{2} / \mathrm{Tb}}\right)+M_{s, \mathrm{Top}}^{\mathrm{Co}}\left(h_{\|}^{\mathrm{Tb} / \mathrm{Co}}-h_{\|}^{\mathrm{Co} / P t}\right)+(n-1)\left(M_{s}^{\mathrm{Co}}+M_{s}^{\mathrm{Tb}}\right)\left(h_{\|}^{\mathrm{Tb} / \mathrm{Co}}-h_{\|}^{\mathrm{Co} / \mathrm{Tb}}\right)\right] / M_{s} .
\end{aligned}
$$

The experimental data obtained in Sec. III indicate that DMI decreases with the number of repetitions, which suggests that DMI is solely due to the Pt/Co interface and absent at $\mathrm{Co} / \mathrm{Tb}$ interfaces $\left(h_{\mathrm{DM}}^{\mathrm{Co} / \mathrm{Tb}}, h_{\mathrm{DM}}^{\mathrm{Tb} / \mathrm{Co}} \approx 0\right)$, so that

$$
H_{\mathrm{DM}}^{\mathrm{eff}} \approx-\frac{M_{s, \mathrm{Top}}^{\mathrm{Co}} h_{\mathrm{DM}}^{\mathrm{Co} / P t}}{(n-1)\left(M_{s}^{\mathrm{Co}}-M_{s}^{\mathrm{Tb}}\right)+M_{s, \text { Top }}^{\mathrm{Co}}-M_{s, \text { Bottom }}^{\mathrm{Tb}}} .
$$

We neglected the contribution of $\mathrm{SiO}_{2} / \mathrm{Tb}$ interfaces due to the weak $\mathrm{SOC}$ of $\mathrm{SiO}_{2}$. In contrast, SOT fields increase with the number of repetitions, which suggests that $\mathrm{SOT}$ fields are present at all the interfaces. Assuming that $\mathrm{SiO}_{2} / \mathrm{Tb}$ interfaces do not produce significant SOT field $\left(h_{D M, \perp, \|}^{\mathrm{SiO}_{2} / \mathrm{Tb}} \approx 0\right)$, the total SOT fields read

$$
\begin{aligned}
h_{\perp}^{\text {eff }} & =\frac{(n-1)\left(M_{s}^{\mathrm{Co}}-M_{s}^{\mathrm{Tb}}\right)\left(h_{\perp}^{\mathrm{Tb} / \mathrm{Co}}-h_{\perp}^{\mathrm{Co} / \mathrm{Tb}}\right)-M_{s, \text { Bottom }}^{\mathrm{Tb}} h_{\perp}^{\mathrm{Tb} / \mathrm{Co}}+M_{s, \mathrm{Top}}^{\mathrm{Co}}\left(h_{\perp}^{\mathrm{Tb} / \mathrm{Co}}-h_{\perp}^{\mathrm{Co} / P t}\right)}{(n-1)\left(M_{s}^{\mathrm{Co}}-M_{s}^{\mathrm{Tb}}\right)+M_{s, \mathrm{Top}}^{\mathrm{Co}}-M_{s, \text { Bottom }}^{\mathrm{Tb}}}, \\
h_{\|}^{\text {eff }} & =\frac{(n-1)\left(M_{s}^{\mathrm{Co}}+M_{s}^{\mathrm{Tb}}\right)\left(h_{\|}^{\mathrm{Tb} / \mathrm{Co}}-h_{\|}^{\mathrm{Co} / \mathrm{Tb}}\right)+M_{s, \text { Bottom }}^{\mathrm{Tb}} h_{\|}^{\mathrm{Tb} / \mathrm{Co}}+M_{s, \mathrm{Top}}^{\mathrm{Co}}\left(h_{\|}^{\mathrm{Tb} / \mathrm{Co}}-h_{\|}^{\mathrm{Co} / P t}\right)}{(n-1)\left(M_{s}^{\mathrm{Co}}-M_{s}^{\mathrm{Tb}}\right)+M_{s, \mathrm{Top}}^{\mathrm{Co}}-M_{s, \text { Bottom }}^{\mathrm{Tb}}} .
\end{aligned}
$$

This simple phenomenological model shows that the thickness dependence of the SOT fields depends on the relative magnitude of the torque at $\mathrm{Co} / \mathrm{Tb}, \mathrm{Tb} / \mathrm{Co}$, and $\mathrm{Co} / \mathrm{Pt}$ interfaces. If the SOT field is dominated by $\mathrm{Tb} / \mathrm{Co}$ and $\mathrm{Co} / \mathrm{Tb}$ interfaces, $h_{\|, \perp}^{\mathrm{Tb} / \mathrm{Co}}-h_{\|, \perp}^{\mathrm{Co} / \mathrm{Tb}} \gg h_{\|, \perp}^{\mathrm{Co} / P t}$, one can expect that the overall SOT effective field increases with the number of repetitions, as observed in Sec. III.

\section{B. DMI and SOT field at $\mathrm{Co} / \mathrm{Tb}$ interfaces}

The analysis provided above implies that DMI is weak at $\mathrm{Co} / \mathrm{Tb}$ interfaces while SOT fields are sizable at these interfaces. This difference can be attributed to the nature of the electron orbitals involved in each mechanism.

DMI is the antisymmetric exchange between local spins and, as such, involves mainly localized electrons. In $\mathrm{Tb}$, the magnetism is carried by $4 f$ orbitals located far below Fermi energy, and therefore hybridizing only weakly to $3 d$ orbitals of Co via $5 d$ orbitals [45]. This is in sharp contrast with $\mathrm{Pt} / \mathrm{Co}$ interfaces where $5 d$ orbitals of Pt couple directly to $3 d$ orbitals of $\mathrm{Co}$, thereby acquiring magnetism by proximity effect. The magnetic coupling being much larger at $\mathrm{Pt} / \mathrm{Co}$ interfaces than at $\mathrm{Tb} / \mathrm{Co}$ interfaces, one can reasonably speculate that DMI should be larger in the former than in the latter.

In contrast with DMI, SOT is a nonequilibrium phenomenon that involves transport electrons at Fermi level. It has been recently showed that the Rashba effect at the surface of Tb layers and its monoxides is quite large [46], which should lead to sizable SOT fields. In addition, since Tb/Co interfaces are not sharp, Tb-Co intermixing is expected. Tb could act as an impurity in Co and one can envisage that it leads to the emergence of extrinsic SHE due to rare-earth impurity-induced skew scattering in the ultrathin TM layer [31,32]. Such a resonant skew scattering results in a giant spin Hall effect, possibly an order of magnitude larger than in $\mathrm{Pt}$ [31,32]. In contrast, the Co impurity in $\mathrm{Tb}$ does not induce extrinsic SHE, which can be only realized in very low resistivity metals. Notice that this effect can only be significant as long as the magnetic exchange between itinerant and local spins is not too strong; otherwise, the itinerant spin remains aligned on the local magnetization.

\section{Compensation between SOT and STT}

To complete our study, we finally discuss the critical thickness where the DW stops moving under the SHE due to the cancellation of the SOTs and bulk STT in the A-, B-, and $\mathrm{C}$-stack wires. Figure 5 shows the DW velocity as a function of current density in the Tb/Co multilayer of A stack and B stack with various $\mathrm{Tb} / \mathrm{Co}$ repetition numbers $(n)$. Seo et al. [8] reported that the magnitude of antidamping SOT is inversely proportional to the thickness of the ferromagnetic (FM) layer. Therefore, the SOT is expected to be compensated by the bulk STT at a critical thickness of the FM layer. We find that the critical thicknesses are $\sim 13.2,9.9$, and $8 \mathrm{~nm}$ for the A, B, and $\mathrm{C}$ stack (not shown), respectively. The thickest magnetic layer to observe the SOT-induced DW motion is the A stack, which has the smallest $J_{\mathrm{c}}$ as shown in Fig. 3(b). This result suggests that the inner $\mathrm{Tb} / \mathrm{Co}$ interfaces play a role for the DW motion in the A stack. As discussed above, DMI is negligible with increasing $n$ as well as the thickness [Fig. 4(b)]. Therefore, DMI should not be strong enough to stabilize the Néel wall 


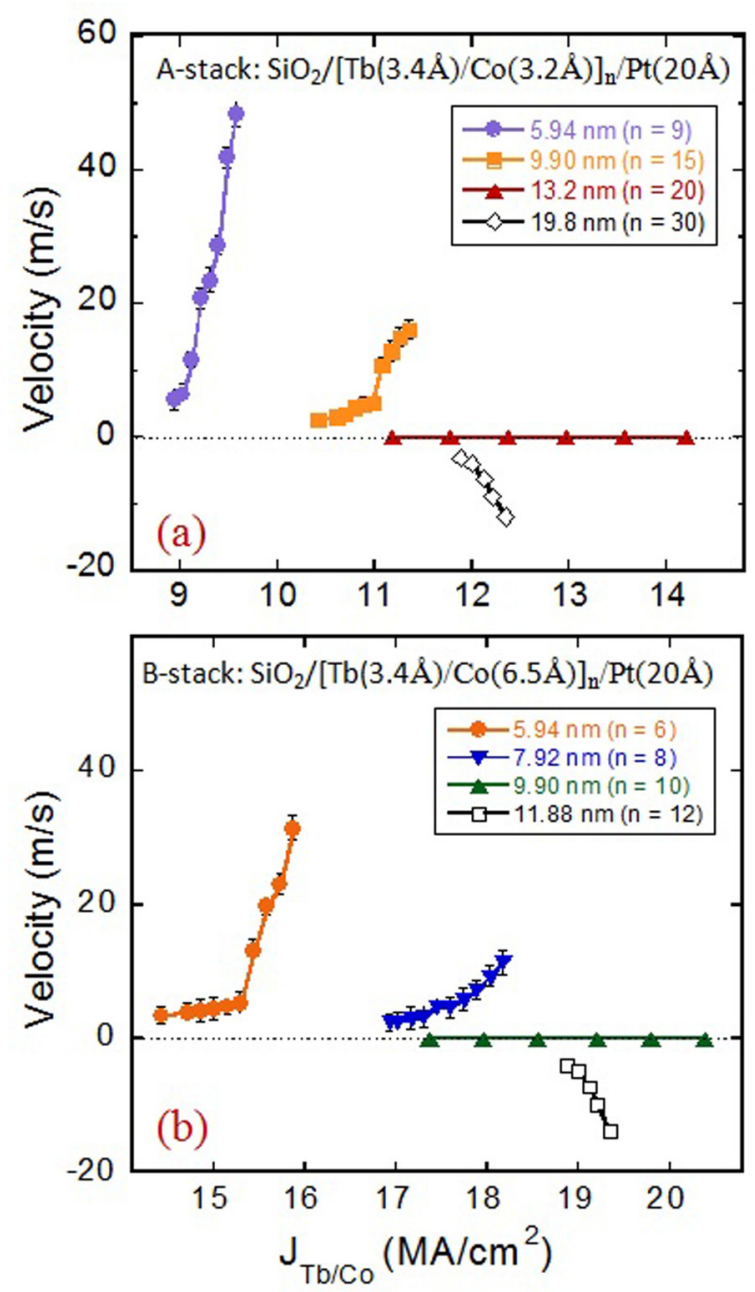

FIG. 5. (a) DW velocity as a function of current density in $\mathrm{Co} / \mathrm{Tb}$ multilayer of (a) A stack and (b) B stack for various $\mathrm{Co} / \mathrm{Tb}$ repetition number $(n)$. structure in thicker samples. In that case, the Bloch DWs would be moved in the direction of conduction electrons due to STT [ $n>20$ in Fig. 5(a)]. A high efficiency of current-induced SOTs has also been reported in $20 \mathrm{~nm}$-thick $\mathrm{Co} / \mathrm{Pd}$ multilayers with perpendicular magnetic anisotropy [43]. The authors concluded that the observed effect could not be explained only by SHE-induced torque at the outer interfaces.

\section{CONCLUSION}

In conclusion, we have studied the current-induced DW motion in the perpendicular magnetized $\mathrm{Tb} / \mathrm{Co}$ wires with different layered structures. The critical current density was found to strongly depend on the layered structures. The lowest critical current density $\sim 1.5 \times 10^{11} \mathrm{~A} / \mathrm{m}^{2}$ and highest slope of DW velocity were obtained for the A-stack wire having thin Co sublayers and more $\mathrm{Tb} / \mathrm{Co}$ interfaces, which suggests that (antidamping) SOT-induced DW motion is enhanced due to contributions from $\mathrm{Tb} / \mathrm{Co}$ interfaces. We suggest that such an enhanced antidamping arises from skew scattering on $\mathrm{Tb}$ rare-earth impurities present in Co sublayers. Our study indicates an efficient way to reduce the critical current density for DW motion through inner interface engineering.

\section{ACKNOWLEDGMENTS}

This work was partially supported by the Ministry of Education, Culture, Sports, Science and Technology, Japan Supported Program for Strategic Research Foundation at Private University (2014-2020) and KAKENHI No. 26630137, and the National Research Foundation (NRF), Prime Minister's Office, Singapore, under its Competitive Research Programme (CRP Award No. NRFCRP12-2013-01). H.Y. thanks the Singapore Spintronics Consortium (SG-SPIN) for support. A.M. acknowledges support from the King Abdullah University of Science and Technology (KAUST).
[1] L. Berger, Phys. Rev. B 54, 9353 (1996).

[2] J. C. Slonczewski, J. Magn. Magn. Mater. 159, L1 (1996).

[3] J. Jaworowicz, N Vernier, J. Ferré, A. Maziewski, D. Stanescu, D. Ravelosona, A. S. Jacqueline, C. Chappert, B. Rodmacq, and B. Diény, Nanotechnol. 20, 215401 (2009).

[4] L. Leem and J. S. Harris, J. Appl. Phys. 105, 07 D102 (2009).

[5] L. Thomas, R. Moriya, C. Rettner, and S. S. P. Parkin, Science 330, 1810 (2010).

[6] I. M. Miron, T. Moore, H. Szambolics, L. D. Buda-Prejbeanu, S. Auffret, B. Rodmacq, S. Pizzini, J. Vogel, M. Bonfim, A. Schuhl, and G. Gaudin, Nature Mater. 10, 419 (2011).

[7] K.-S. Ryu, L. Thomas, S. H. Yang, and S. S. P. Parkin, Appl. Phys. Express 5, 093006 (2012).

[8] S.-M. Seo, K.-W. Kim, J. Ryu, H.-W. Lee, and K.-J. Lee, Appl. Phys. Lett. 101, 022405 (2012).

[9] S. Emori, U. Bauer, S.-M. Ahn, E. Martinez, and G. S. D. Beach, Nat. Mater. 12, 611 (2013).

[10] T. Koyama, H. Hata, K.-J. Kim, T. Moriyama, H. Tanigawa, T. Suzuki, Y. Nakatani, D. Chiba, and T. Ono, Appl. Phys. Express 6, 033001 (2013).
[11] X. Qiu, K. Narayanapillai, Y. Wu, P. Deorani, D.-H. Yang, W.-S. Noh, J.-H. Park, K.-J. Lee, H.-W. Lee, and H. Yang, Nature Nanotechnol. 10, 333 (2015).

[12] W. Legrand, R. Ramaswamy, R. Mishra, and H. Yang, Phys. Rev. Appl. 3, 064012 (2015).

[13] S. Zhang, Phys. Rev. Lett. 85, 393 (2000).

[14] L. Liu, C.-F. Pai, Y. Li, H. W. Tseng, D. C. Ralph, and R. A. Buhrman, Science 336, 555 (2012).

[15] D. Bang and H. Awano, Jpn. J. Appl. Phys. 52, 123001 (2013).

[16] P. M. Haney, H.-W Lee, K.-J. Lee, A. Manchon, and M. D. Stiles, Phys. Rev. B 88, 214417 (2013).

[17] X. Qiu, P. Deorani, K. Narayanapillai, K.-S. Lee, K.-J. Lee, H.-W. Lee, and H. Yang, Sci. Rep. 4, 4491 (2014).

[18] X. Wang and A. Manchon, Phys. Rev. Lett. 108, 117201 (2012).

[19] P. M. Haney, H.-W. Lee, K.-J. Lee, A. Manchon, and M. D. Stiles, Phys. Rev. B 87, 174411 (2013).

[20] F. Freimuth, S. Blügel, and Y. Mokrousov, Phys. Rev. B 90, 174423 (2014).

[21] T. Moriya, Phys. Rev. 120, 91 (1960).

[22] I. E. Dzyaloshinskii, Sov. Phys. JETP 19, 1163 (1964). 
[23] M. Heide, G. Bihlmayer, and S. Blügel, Phys. Rev. B 78, 140403(R) (2008).

[24] A. V. Khvalkovskiy, V. Cros, D. Apalkov, V. Nikitin, M. Krounbi, K. A. Zvezdin, A. Anane, J. Grollier, and A. Fert, Phys. Rev. B 87, 020402(R) (2013).

[25] K.-S. Ryu, L. Thomas, S.-H. Yang, and S. S. P. Parkin, Nature Nanotechnol. 8, 527 (2013).

[26] O. Boulle, S. Rohart, L. D. Buda-Prejbeanu, E. Jué, I. M. Miron, S. Pizzini, J. Vogel, G. Gaudin, and A. Thiaville, Phys. Rev. Lett. 111, 217203 (2013).

[27] K.-S. Ryu, S.-H. Yang, L. Thomas, and S. S. P. Parkin, Nature Commun. 5, 3910 (2014).

[28] K. Di, V. L. Zhang, H. S. Lim, S. Choon Ng, M. H. Kuok, J. Yu, J. Yoon, X. Qiu, and H. Yang, Phys. Rev. Lett. 114, 047201 (2015).

[29] K. Ueda, K.-J. Kim, Y. Yoshimura, R. Hiramatsu, T. Moriyama, D. Chiba, H. Tanigawa, T. Suzuki, E. Kariyada, and T. Ono, Appl. Phys. Express 7, 053006 (2014).

[30] D. Bang and H. Awano, J. Appl. Phys. 117, 17 D916 (2015).

[31] A. Fert and A. Friederich, Phys. Rev. B 13, 397 (1976).

[32] T. Tanaka and H. Kontani, New J. Phys. 11, 013023 (2009).

[33] A. Moore, I. M. Miron, G. Gaudin, G. Serret, S. Auffret, B. Rodmacq, A Schuhl, S. Pizzini, J. Vogel, and M. Bonfim, Appl. Phys. Lett. 93, 262504 (2008).

[34] M. Nawate, T. Morikawa, S. Tsunashima, and S. Uchiyama, IEEE Trans. Magn. 26, 2739 (1990).
[35] L. Ertl, G. Endl and H. Hoffmann, J. Magn. Magn. Mater. 113, 227 (1992).

[36] K.-J. Kim, R. Hiramatsu, T. Moriyama, H. Tanigawa, T. Suzuki, E. Kariyada, and T. Ono, Appl. Phys. Express 7, 053003 (2014).

[37] P. P. J. Haazen, E. Mure, J. H. Franken, R. Lavrijsen, H. J. M Swagten, and B. Koopsman, Nature Mater. 12, 299 (2013).

[38] A. Thiaville, S. Rohart, É. Jué, V. Cros, and A. Fert, Europhys. Lett. 100, 57002 (2012).

[39] E. Martinez, S. Emori, and G. S. D. Beach, Appl. Phys. Lett. 103, 072406 (2013).

[40] A. Hrabec, N. A. Porter, A. Wells, M. J. Benitez, G. Burnell, S. McVitie, D. McGrouther, T. A. Moore, and C. H. Marrows, Phys. Rev. B 90, 020402(R) (2014).

[41] J. Kim, J. Sinha, M. Hayashi, M. Yamanouchi, S. Fukami, T. Suzuki, S. Mitani, and Ohno, Nature Mater. 12, 240 (2013).

[42] K. Garello, I. M. Miron, C. O. Avci, F. Freimuth, Y. Mokrousov. S. Blügel, S. Auffret, O. Boulle, G. Gaudi, and P. Gambardella, Nature Nanotechnol. 8, 587 (2013).

[43] M. Jamali, K. Narayanapillai, X. Qiu, L. M. Loong, A. Manchon, and H. Yang, Phys. Rev. Lett. 111, 246602 (2013).

[44] H. R. Lee, K. Lee, J. Cho, Y. H. Choi, C. Y. You, M. H. Jung, F. Bonell, Y. Shiota, S. Miwa, and Y. Suzuki, Sci. Rep. 4, 6548 (2014).

[45] I. A. Campbell, J. Phys. F: Metal Phys. 2, L47 (1972).

[46] O. Krupin, G. Bihlmayer, K. M. Döbrich, J. E. Prieto, K. Starke, S. Gorovikov, S. Blügel, S. Kevan, and G. Kaindl, New J. Phys. 11, 013035 (2009). 